\title{
COMPÓSITOS REFORÇADOS COM FIBRAS LONGAS DE BAMBU: MODIFICAÇÃO SUPERFICIAL E ANÁLISE MECÂNICA*
}

\author{
Ana Beatriz Ferreira Sousa ${ }^{1}$ \\ Santino Loruan Silvestre $\mathrm{Melo}^{2}$ \\ Laudenor Amorim ${ }^{3}$ \\ Enio Pontes de Deus ${ }^{4}$
}

\section{Resumo}

Compósitos são hoje uma importante vertente para o desenvolvimento de produtos com propriedades específicas. Quando compósitos são produzidos a partir de materiais renováveis e biodegradáveis, estes se tornam sustentáveis, devido à sua fonte de matéria prima. As fibras naturais vegetais são abundantes em todo o mundo e responsáveis pelas propriedades mecânicas das plantas, que representam um potencial alvo de estudo no desenvolvimento de materiais "verdes". Baseado nisso, o objetivo desse trabalho é produzir compósitos de matriz polimérica termoplástica reciclada com fibras naturais de bambu longas, provenientes de biomassa regional, e analisar a influência das fibras como reforço nos compósitos. Realizaram-se os processos de lavagem, mercerização e acetilação para tratar quimicamente as fibras. Os resultados, quando comparados com a literatura disponível, indicaram que o compósito reforçado com as fibras longas de bambu modificadas apresentou melhores propriedades mecânicas que sua matriz polimérica. Por consequente, constata-se que se obteve êxito a partir das técnicas utilizadas.

Palavras-chave: Fibras de Bambu; Compósitos; Tratamentos Químicos; Ensaio de Tração.

\section{COMPOSITES REINFORCED WITH LONG BAMBOO FIBERS: SUPERFICIAL MODIFICATION AND MECHANICAL ANALYSIS}

\begin{abstract}
Composites are an important aspect for the development of products with specific properties. When composites are produced from renewable and biodegradable materials, they become sustainable due to their source of raw material. Natural vegetable fibers are abundant throughout the world and responsible for the mechanical properties of plants, which represent a potential target of study in the development of "green" materials. Based on this, the objective of this work is to produce composites of recycled thermoplastic polymer matrix with long bamboo natural fibers, from regional biomass, and analyse the influence of fibers as reinforcement in composites. The processes of washing, mercerization and acetylation were conducted to chemically treat the fibers. The results, when compared with the available literature, indicated that the reinforced composite with long modified bamboo fibers presented better mechanical properties than the polymer matrix. Consequently, it was noted that the techniques was used with successful.

Keywords: Bamboo Fibers; Composites; Chemical Treatment; Traction Trial.

1 Engenharia Metalúrgica e de Materiais, Graduanda, Laboratório de Mecânica da Fratura e da Fadiga/Departamento de Engenharia de Engenharia Metalúrgica e de Materiais, Universidade Federal do Ceará, Fortaleza, Ceará, Brasil.

2 Físico Bacharel/Licenciado, Doutorando, Professor Mestre, FAFIDAM, Universidade Estadual do Ceará, Limoeiro do Norte, Ceará, Brasil.

3 Químico Licenciado, Mestrando, Laboratório de Mecânica da Fratura e da Fadiga/Departamento de Engenharia de Engenharia Metalúrgica e de Materiais, Fortaleza, Ceará, Brasil.

4 Engenheiro Civil, Doutor, Professor Doutor, Laboratório de Mecânica da Fratura e da Fadiga/Departamento de Eng. Metalúrgica e de Materiais, Fortaleza, Ceará, Brasil.
\end{abstract}




\section{INTRODUÇÃO}

Em virtude da necessidade de novas tecnologias, o domínio de novos materiais acompanha a evolução humana através dos séculos - a celulose e a seda são exemplos disso. Então, a relevância dos polímeros surge da necessidade de produzir materiais como borrachas, plásticos e fibras, que tenham aplicações cada vez mais específicas [1]. Por sua vez, os materiais compósitos, que têm suas propriedades bem definidas, possuem uma demanda mais específica que a dos polímeros. Sendo assim, a utilização desses materiais oferece benefícios econômicos e ambientais.

Em relação aos materiais convencionalmente utilizados, as fibras naturais apresentam boas propriedades mecânicas. A maioria das fibras naturais tem como principal desvantagem a variação das propriedades mecânicas conforme seção transversal e longitudinal. Entretanto, as fibras de bambu utilizadas nesse trabalho, da espécie Bambusa Vulgaris, dipõem da vantagem de uma orientação uniforme na seção longitudinal do colmo [2].

O objetivo deste trabalho é avaliar as propriedades de resistência e módulo de elasticidade dos materiais utilizados para a produção dos compósitos e seus respectivos corpos de prova. Ademais, visa-se verificar a compatibilidade do Polietileno Reciclado com as fibras naturais modificadas através dos tratamentos químicos de lavagem, merceirização e aceitilação. Para isso, realizaram-se ensaios de tração nos corpos de prova.

\section{MATERIAIS E MÉTODOS}

\subsection{Fibras de Bambu}

As propriedades mecânicas das fibras do bambu se devem à sua estrutura LCC (lignincarbohydrate complex), onde microfibrilas de cristais de celulose ligadas por hemicelulose são envoltas em uma matriz amorfa de lignina e hemicelulose. $O$ bambu atinge sua idade adulta aproximadamente aos 3 anos e pode suportar uma temperaturas de $40^{\circ} \mathrm{C}$ à $50^{\circ} \mathrm{C}$.

O Bambusa Vulgaris, objeto de estudo desse trabalho, é cultivados espontaneamente em larga escala na região Nordeste do Brasil. É constituído basicamente de colmo, que abrigam os nós, entrenós e a base do caule. O colmo cresce linearmente em forma cilíndrica e, quando na idade adulta, pode atingir entre $8 \mathrm{~m}$ e $20 \mathrm{~m}$ de altura, com diâmetro extrerno variando de 5 à $10 \mathrm{~cm}$ [3]. Na Fig. 1, apresenta-se uma imagem do bambu e seus constituintes externos. 

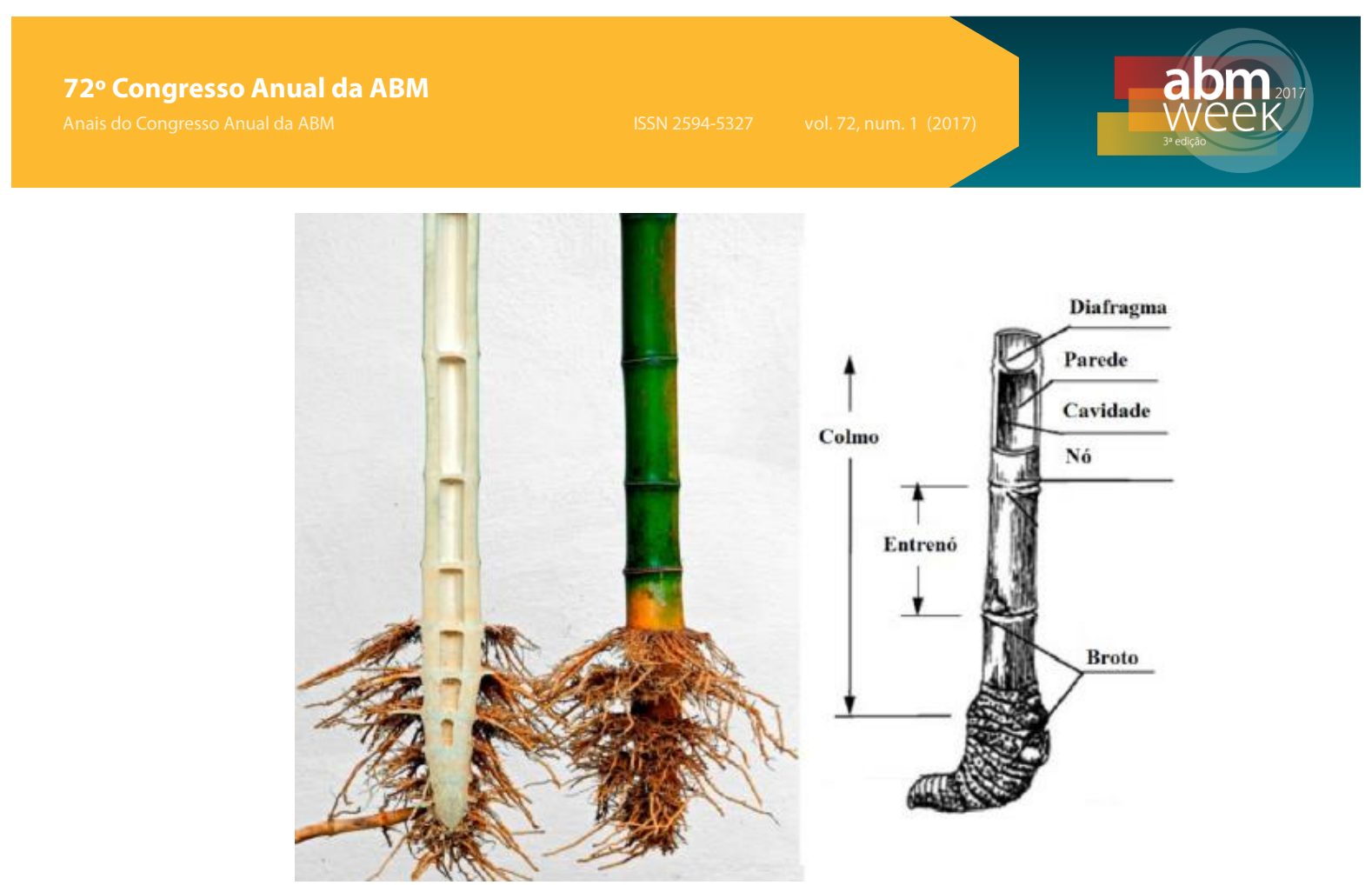

Figura 1. Bambu e seus constituintes externos [4].

\subsection{Procedimento Experimental}

\subsubsection{Coleta do Bambu}

Neste trabalho, foram coletados bambus presentes no Campus Pici da Universidade Federal do Ceará, com idade adulta — prevista pela observação da coloração amarelo/esverdeada da casca -, com aproximadamente 3,5 m de altura. Os colmos mediam aproximadamente $4,5 \mathrm{~cm}$ de diâmetro interno e $9 \mathrm{~cm}$ de diâmetro interno. A coleta ocorreu em março de 2017 e o material foi instantaneamente levado para os processos que serão explanados posteriormente.

\subsubsection{Preparação e separação das amostras do Bambu}

O colmo extraído foi levado para o Laboratório de Mecânica da Fratura e da Fadiga (LAMEFF), no Centro de Tecnologia no Campus do Pici. Para a separação dos nós e internós, utilizou-se uma serra fita automática. Os nós foram descartados e os internós seguiram para a retirada da casca (lâminas transversais de $1 \mathrm{~mm}$ de espessura), que foi descartada.

Após isso, os internós foram laminados transversalmente em cavacos de 2 $\mathrm{mm}$, ao meio do diâmetro interno e externo, garantindo que o material com a maior quantidade de fibras fosse selecionado, visto que a região mais interna possui uma quantidade de fibras reduzida. À vista disto, o rendimento aproximado de fibras do colmo do bambu foi de $50 \%$, pois a casca e região mais interna, onde se encontra a presença de mais lignina do que de fibras, foram descartadas.

Todo o processo até a separação dos cavacos foi feito antes que a umidade natural do bambu fosse perdida, porque a laminação é dificultada de acordo com o tempo em que o material se torna mais enxuto. As fibras contínuas foram retiradas manualmente dos cavacos transversais, em um processo possibilitado devido à orientação regular dos feixes microfibrilares da região selecionada. 


\subsubsection{Tratamento Químico}

O foco desse trabalho é a utilização das fibras naturais vegetais como agente de carga e reforço em compósitos termoplásticos, o que exige compatibilidade química entre seus componentes [5]. A compatibilidade entre as fibras naturais e outras fases depende da inserção de grupos químicos ou retirada de constituintes das fibras, de acordo com as propriedades finais que se deseja obter [6].

\subsubsection{Processo de lavagem}

Esta etapa consistiu na submersão das fibras em água pura à $80^{\circ} \mathrm{C}$ durante uma hora. Após isso, estas foram postas em água corrente durante 5 min e secadas em uma estufa à $60^{\circ} \mathrm{C}$. Esse processo foi realizado sempre intercalado ao final dos demais processos de mercerização e acetilação. De fato, tornou-se de suma importância para a observação da eficiência do tratamento químico das fibras, já que possibilitou, pela coloração final da água, uma suposição da quantidade de lignina liberada.

\subsubsection{Processo de mercerização}

As fibras de bambu contínuas foram imersas, à $60^{\circ} \mathrm{C}$ e durante uma hora, em solução de $\mathrm{NaOH}$ na concentração de $2 \%$. Esse procedimento foi repetido duas vezes. Ao final do procedimento, as fibras passaram pelo processo de lavagem até atingirem um $\mathrm{pH}=7$.

\subsubsection{Processo de acetilação}

Após os procedimentos de limpeza e hidrólise, as fibras foram intumescidas em uma solução - na proporção de $1 / 10$ e, assim como no processo de mercerização, à $60^{\circ} \mathrm{C}$ durante uma hora - de anidrido acético e ácido acético $(2,0$ : 1,0 em massa). Nesta na mistura, ainda foram adicionadas 5 gotas de ácido sulfúrico para cada $500 \mathrm{ml}$ de solução.

\subsection{Polietileno}

Neste trabalho, utilizou-se o Polietileno Misto Reciclado (PMR) como matéria prima base. O polietileno pode ter diversas aplicações, mas uma das principais é a indústria de embalagens flexíveis. E é a indústria de alimentos a maior consumidora de embalagens flexíveis, absorvendo $35 \%$ do total dessas embalagens produzidas, segundo dados de 2013 da Associação Brasileira da Indústria de Embalagens Plásticas Flexíveis (ABIEF). Geralmente, também é usado para isolamento em condutores de correntes elétricas, tubos e próteses.

O polietileno foi sintetizado pela primeira vez pelo químico alemão Hans von Pechmann. Eugen Bamberger e Friedrich Tschirner o caracterizaram e descobriram grandes cadeias compostas por $-\mathrm{CH}_{2-}$, de onde deriva seu nome. É um polímero parcialmente cristalino, flexível, cujas propriedades são acentuadamente influenciadas pela quantidade relativa das fases amorfa e cristalina [7]. 


\subsection{Montagem da placa}

Compósitos com fibras contínuas e orientadas, de matriz polimérica termoplástica, ainda não possuem um método de produção em massa. A forma de produção desses compósitos, em escala laboratorial utilizado nesse trabalho é o método de confecção por compressão, com pressão e temperatura controladas. Um molde de Al-Mg para a produção de placas compósitas foi então elaborado para a confecção desses materiais.

As placas de polietileno misto reciclado foram confeccionadas considerando sua densidade. Sendo assim, pesou-se quantidade suficiente para a confecção de uma placa de $260 \mathrm{~mm}$ de diâmetro e $80 \mathrm{~mm}$ de largura e até $7 \mathrm{~mm}$ de espessura. $\mathrm{O}$ material, na forma de pellets, foi disperso no molde e aquecido a $180^{\circ} \mathrm{C}$. Após a fundição do polietileno reciclado, as fibras foram adicionadas de forma orientada longitudinalmente e perpendicularmente à placa, para que corpos de prova pudessem ser obtidos a $0^{\circ}$ ou $90^{\circ}$.

O molde, então, foi fechado e o fluxo de calor cessado, introduzindo uma pressão moderada para que o material, ainda em estado líquido, não escapasse pelas laterais. Aproximadamente em $120^{\circ} \mathrm{C}$, a pressão máxima foi acionada, sendo mantida assim até que a temperatura ambiente fosse atingida (momento da retirada da placa).

\section{RESULTADOS E DISCUSSÃO}

A placa foi subdividida em seis (6) corpos de prova com dimensões apresentadas na Tabela 1 - adaptadas da norma ASTM 3039, tal que diz respeito aos corpos de prova orthotropic, que possuem propriedades diferentes de acordo com suas direções ortogonais. De fato, o ideal era que os corpos de prova apresentassem dimensões iguais, porém ocorreu uma pequena variação causada pela imprecisão do instrumento de corte utilizado.

Tabela 1. Dimensões dos corpos de prova.

\begin{tabular}{cccc}
\hline NÚMERO & $\begin{array}{c}\text { COMPRIMENTO } \\
(\mathbf{c m})\end{array}$ & $\begin{array}{c}\text { LARGURA } \\
(\mathbf{c m})\end{array}$ & $\begin{array}{c}\text { ESPESSURA } \\
(\mathbf{c m})\end{array}$ \\
\hline 1 & 10,25 & 2,40 & 0,55 \\
\hline 2 & 10,30 & 2,40 & 0,50 \\
\hline 3 & 10,20 & 2,35 & 0,55 \\
\hline 4 & 10,30 & 2,40 & 0,53 \\
\hline 6 & 10,20 & 2,35 & 0,50 \\
\hline 0 & 10,25 & 2,40 & 0,55 \\
\hline
\end{tabular}

Os tamanhos dos corpos de prova variaram apenas alguns milímetros.

Os ensaios foram realizados em maio de 2017 no Laboratório de Ensaios Mecânicos do Institudo Federal de Educação, Ciência e Tecnologia do Ceará (IFCE), Campus Fortaleza. O equipamento utilizado foi a Máquina de Ensaio de Tração de 200 toneladas Sling/Hidráulico Universal. 
Os ensaios foram realizados através da aplicação de uma força ao corpo de prova nas condições controladas. Têm-se que, geralmente, a deformação se dá em velocidade constante até a fratura. Neste caso, uma tensão máxima uniforme de $100 \mathrm{KN}$ foi aplicada ao longo das peças à uma velocidade de $5 \mathrm{~mm} / \mathrm{min}$. Ao final, os corpos de prova apresentaram uma deformação plástica visível nas Figuras 2 e 3.

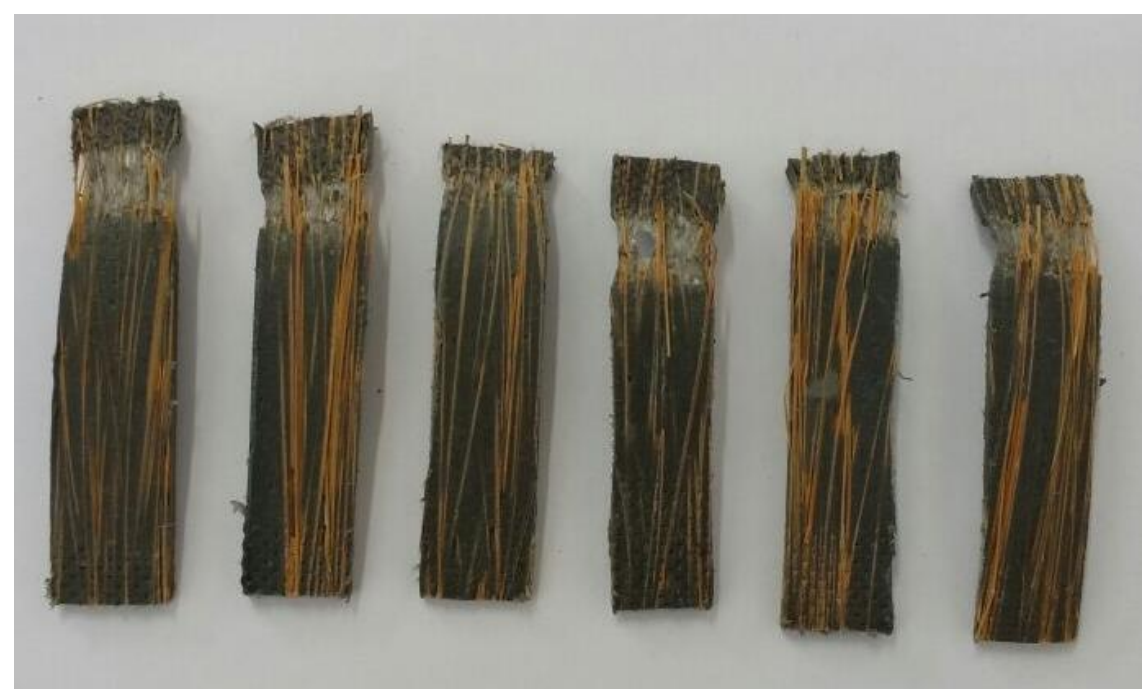

Figura 2. Vista frontal dos corpos de prova após o ensaio de tração. Fonte: Autor.

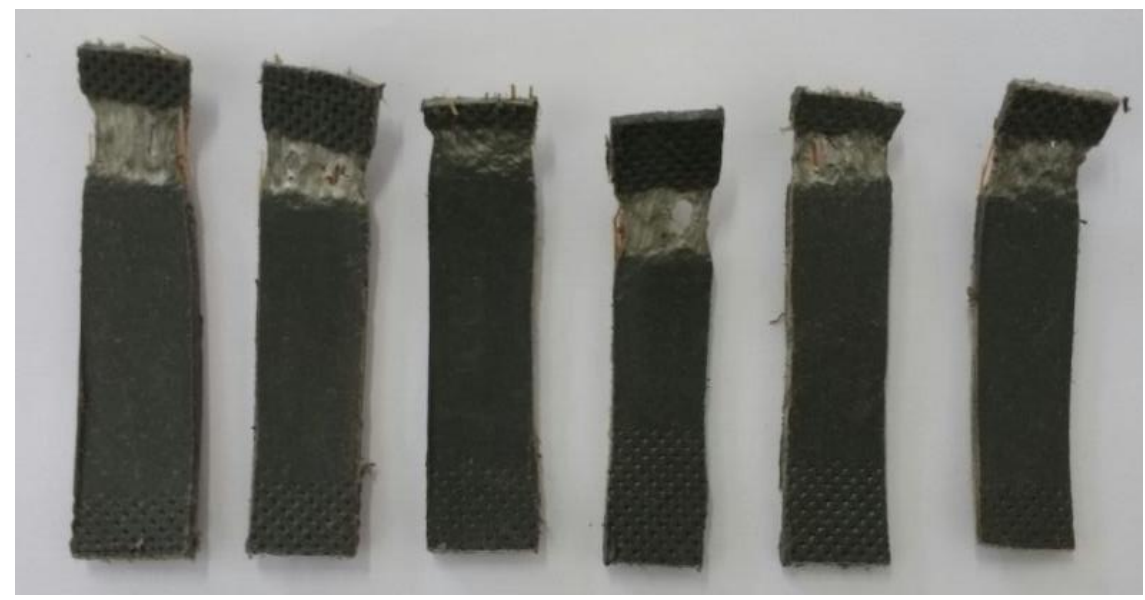

Figura 3. Vista posterior dos corpos de prova após o ensaio de tração. Fonte: Autor. 
No experimento, a tensão suportada pelo material e sua deformação imposta são registradas. Então, os principais dados revelados pelos ensaios foram a tensão máxima na ruptura, tensão de escoamento e o módulo de elasticidade. Estes são apresentados na Tabela 2.

Tabela 2. Resultados do Ensaio de Tração do Polietileno Misto Reciclado.

\begin{tabular}{cccc}
\hline NÚMERO & $\begin{array}{c}\text { MÓDULO DE } \\
\text { ELASTICIDADE } \\
\text { (GPa) }\end{array}$ & $\begin{array}{c}\text { LIMITE DE } \\
\text { ESCOAMENTO } \\
\text { (MPa) }\end{array}$ & $\begin{array}{c}\text { TENSÃO } \\
\text { MÁXIMA } \\
\text { (MPa) }\end{array}$ \\
\hline 1 & 3 & 9 & 19 \\
\hline 2 & 5 & 8 & 18 \\
\hline 3 & 3 & 9 & 16 \\
\hline 4 & 1 & 6 & 13 \\
\hline 5 & 2 & 9 & 20 \\
\hline 6 & 2 & 10 & 22 \\
\hline MÉDIA & $2,66 \pm 1,36$ & $8,5 \pm 1,37$ & $18 \pm 3,16$ \\
\hline
\end{tabular}

Nos ensaios de resistência à tração, a solicitação é feita através de uma deformação por tração e a resposta obtida é um gráfico Tensão versus Deformação. Observe a Figura 4, que mostra o este gráfico composto das seis curvas, cada qual representando seu respectivo corpo de prova. 


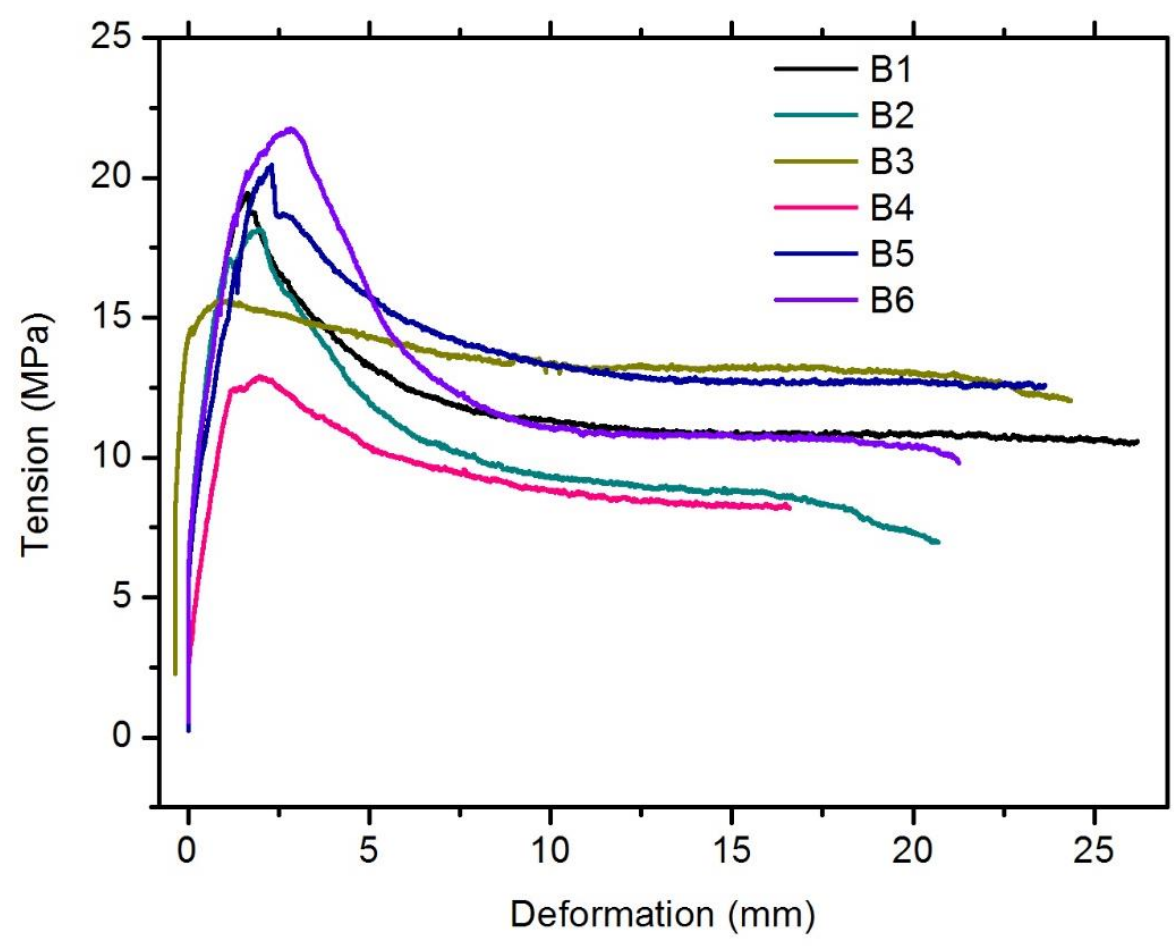

Figura 4. Gráfico da Tensão versus Deformação dos corpos de prova.

Em comparação com a literatura disponível, resultado de outros trabalhos científicos direcionados relacionados ao objeto de estudo deste, obteve-se a Tabela 3 , que reúne dados das propriedades mecânicas de uma placa composta somente por polietileno misto reciclado.

Tabela 3. Resultados do Ensaio de Tração do Polietileno Misto Reciclado.

\begin{tabular}{cccc}
\hline NÚMERO & $\begin{array}{c}\text { MÓDULO DE } \\
\text { ELASTICIDADE } \\
(\mathbf{M P a})\end{array}$ & $\begin{array}{c}\text { LIMITE DE } \\
\text { ESCOAMENTO } \\
(\mathbf{M P a})\end{array}$ & $\begin{array}{c}\text { TENSÃO } \\
\text { MÁXIMA }\end{array}$ \\
\hline 1 & 567,7 & 2 & 5,9 \\
\hline 2 & 496 & 1,7 & 5,1 \\
\hline 3 & 495,3 & 2,13 & 5,9 \\
\hline 4 & 589,4 & 2,4 & 6,1 \\
\hline 5 & 407,3 & 1,8 & 5,2 \\
\hline MÉDIA & $511,14 \pm 32,02$ & $2,01 \pm 0,118$ & $5,64 \pm 0,31$ \\
\hline Propriedades mecâncias de uma placa feita inteiramente com PMR [8].
\end{tabular}

Fazendo uma análise entre a Tabela 2 e a Tabela 3, percebe-se claramente que as propriedades mecânicas do compósito de polietineo misto reciclado reforçado com fibras longas de bambu tratadas quimicamente pelos processos de lavagem, merceirização e aceilação, foram otimizadas.

\section{CONCLUSÃo}


Após a realização dos ensaios de tração e análise dos dados obtidos, concluise que o objetivo deste trabalho foi alcançado. Efetuou-se a avaliação das propriedades de resistência e módulo de elasticidade dos materiais utilizados para a produção dos compósitos. Os resultados foram satisfatórios e reforçaram os dados bibliográficos já existentes no mesmo âmbito de estudo, mesmo que a forma do tratamento das fibras tenha sido alterada. O módulo de elasticidade do composito foi muito maior que o da matriz polimérica e isso que implica a eficiência dos tratamentos químicos utilizados.

Ademais, verificou-se, por meio da observação do resultado final da placa, após o resfriamento até a temperatura ambiente, a compatibilidade do Polietileno Misto Reciclado com as fibras longas de bambu modificadas através dos processos de lavagem, merceirização e aceitilação. Entende-se, então, que a produção de compósitos por compressão tornou-se eficiente em pequena escala. A remoção de componentes poliméricos, como a lignina e a hemicelulose, e modificação química da estrutura superficial com a introdução dos grupos acetila, causaram maior exposição da estrutura da celulose, resultando em materiais com maior compatibilidade química. Cerca de $10 \%$ das fibras foram incorporadas pela matriz polimérica.

\section{REFERÊNCIAS}

1 Hage Jr. Elias. Aspectos históricos sobre o desenvolvimento da ciência e tecnologia de polímeros. Universidade Federal de São Carlos, 1998.

2 Flamínio Levi Neto e Pardini Luis Claudio. Compósitos Estruturais: Clência e Tecnologia (1 ed.). São Paulo: Edgard Blucher, 2006.

3 Wahab. Relationship between physical, anatomical and strength properties of 3-yearold cultivated tropical bamboo gigantochloa scortechinii. Journal of Agricultural and Biological Science. 2012;7(10):782-791.

4 Loruan Silvestre De Melo S. Investigação das Propriedades Mecânicas e Adesão Interfacial dos Compósitos de Polipropileno Virgem e Reciclado Reforçados com Fibras e Microfibras de Bambu [Mestrado]. Universidade Federal do Ceará; 2016.

5 Jahan. Comparative Study of Physical and Elastic Properties of Jute and Glass Fiber Reinforced Ldpe Composites. International Journal of Scientific \& Technology Research, 2012. 68 - 72.

6 Belgacem. Monomers, Polymers and Composites from Renewable Resources. Amsterdã: Elsevier's Science \& Technology. 2008.

7 Fernanda M. B. Coutinho, Ivana L. Mello, Luiz C. de Santa Maria. Polietileno: Principais Tipos, Propriedades e Aplicações. Instituto de Química, UERJ, 2003.

8 Luis De Moura Paiva Júnior Sérgio. Estudo Do Processo De Reciclagem Do Polietileno Para A Produção De Blendas. [Graduação]. Universidade Federal do Ceará, 2017. 